\title{
Protective effect of selegiline on cigarette smoke-induced oxidative stress and inflammation in rat lungs in vivo
}

\author{
Yuting Cui ${ }^{1 \#}$, Kenneth W. K. Liu ${ }^{1,2}$, Mary S. M. Ip ${ }^{1,2}$, Yingmin Liang ${ }^{1}$, Judith C. W. Mak ${ }^{1,2,3}$ \\ ${ }^{1}$ Department of Medicine, The University of Hong Kong, Hong Kong, China; ${ }^{2}$ Research Centre of Heart, Brain, Hormone and Healthy Aging, The \\ University of Hong Kong, Hong Kong, China; 3Pharmacology \& Pharmacy, The University of Hong Kong, Hong Kong, China \\ Contributions: (I) Conception and design: Y Cui, KWK Liu, JCW Mak; (II) Administrative support: MSM Ip, JCW Mak; (III) Provision of study \\ materials or patients: Y Cui, Y Liang; (IV) Collection and assembly of data: Y Cui, KWK Liu, Y Liang; (V) Data analysis and interpretation: Y Cui, \\ Y Liang, JCW Mak; (VI) Manuscript writing: All authors; (VII) Final approval of manuscript: All authors. \\ Correspondence to: Dr. Judith C. W. Mak. Departments of Medicine and Pharmacology \& Pharmacy, The University of Hong Kong, Hong Kong, \\ China. Email: judithmak@hku.hk.
}

Background: Cigarette smoke (CS)-induced build-up of oxidative stress is the leading cause of chronic obstructive pulmonary disease (COPD). Monoamine oxidases (MAOs) are novel sources of reactive oxygen species (ROS) due to the production of hydrogen peroxide $\left(\mathrm{H}_{2} \mathrm{O}_{2}\right)$. However, it remains unclear whether MAO signaling is involved in CS-induced oxidative stress in vivo. This study aimed at investigating the impact of selegiline, a selective MAO-B inhibitor, on CS-induced lung oxidative stress and inflammation in vivo and its underlying mechanism.

Methods: Sprague Dawley rats were randomly divided into four groups: saline plus sham air (Saline/air), saline plus cigarette smoke (Saline/CS), selegiline plus sham air (Slg/air) and selegiline plus cigarette smoke (Slg/CS). Rats from Saline/air and Saline/CS groups were intraperitoneally injected with saline $(2 \mathrm{~mL} / \mathrm{kg}$ body weight) while rats from Slg/air and Slg/CS groups were injected with selegiline ( $2 \mathrm{mg} / \mathrm{kg}$ body weight) about $30 \mathrm{~min}$ prior to exposure daily. The Saline/air and Slg/air groups were exposed to atmospheric air while the Saline/CS and Slg/CS groups were exposed to mainstream CS generated from the whole body inExpose smoking system (SCIREQ, Canada) for twice daily (each for 1 hour with 20 cigarettes). After 7 days, rats were sacrificed to collect bronchoalveolar lavage (BAL) and lung tissues for the measurement of oxidative/anti-oxidative and inflammatory/anti-inflammatory makers respectively.

Results: CS caused significant elevation of MAO-B activity, reduction of total antioxidant capacity (T-AOC) and rGSH/GSSG ratio, and enhancement of superoxide dismutase (SOD) activity in rat lung. Selegiline significantly only reversed CS-induced elevation of MAO-B activity and reduction of rGSH/GSSG ratio. The CS-induced elevation of heme oxygenase-1 (HO-1) and $\mathrm{NAD}(\mathrm{P}) \mathrm{H}$ quinone dehydrogenase 1 (NQO1) expression via nuclear factor erythroid 2-related factor 2 (Nrf2) was also reversed by selegiline. Despite of CSinduced increase in total cell counts, especially the number of macrophages, selegiline had no effect. Selegiline attenuated CS-induced elevation of pro-inflammatory mediators (CINC-1, MCP-1 and IL-6) and restored CSinduced reduction of anti-inflammatory mediator IL-10 in BAL, which was driven through MAPK and NF- $\kappa$ B. Conclusions: Inhibition of MAO-B may provide a promising therapeutic strategy for CS-mediated oxidative stress and inflammation in acute CS-exposed rat lungs.

Keywords: Cigarette smoke (CS); inflammation; monoamine oxidase-B; oxidative stress; selegiline

Submitted Mar 12, 2020. Accepted for publication Aug 28, 2020.

doi: $10.21037 /$ atm-20-2426

View this article at: http://dx.doi.org/10.21037/atm-20-2426

\footnotetext{
${ }^{\#}$ Present address: College of Life Sciences, Shandong University of Technology, Zibo, China
} 


\section{Introduction}

Chronic obstructive pulmonary disease (COPD), which is characterized by progressively irreversible airflow limitation and chronic airway inflammation, has been estimated to be the third leading cause of mortality by 2020 (1-3). Cigarette smoke (CS) is thought to be the primary cause of COPD, and a key inducer of reactive oxygen species (ROS) in the respiratory tract. Increase in ROS from endogenous and exogenous sources without sufficient elimination from endogenous antioxidant defense system (enzymatic and non-enzymatic antioxidants) leading to cellular oxidative stress, is believed to play important roles in many facets of cellular functions including inflammation, mitochondrial dysfunction and onset of apoptosis (4-6).

Among the many endogenous ROS sources, mitochondria are perhaps the best one being characterized. Leakage of electrons from the respiratory chain as well as other $\mathrm{H}_{2} \mathrm{O}_{2}$-generating enzymes residing in the mitochondria make them important endogenous ROS sites in the cells (7). Monoamine oxidases (MAOs), which are bound to the outer mitochondrial membrane, are well known for the regulation of neurotransmission of monoamines. Two types of MAOs (MAO-A and -B) with different substrate and inhibitor specificity have been identified in a previous study (8), which generate $\mathrm{H}_{2} \mathrm{O}_{2}$ as byproducts during degradation of a variety of monoamines.

It has been demonstrated that mitochondria may be a potential therapeutic target for airway diseases $(4,9)$; therefore investigating the effects of mitochondrialbound $\mathrm{H}_{2} \mathrm{O}_{2}$-generating enzyme MAOs may be of interest. Our group has previously demonstrated the elevation of MAO-B activity in airway epithelial cells, which was inhibited by a selective MAO-B inhibitor, selegiline in vitro (10). However, whether inhibition of MAO could reverse CS-induced oxidative stress and inflammation has not been addressed in vivo. Indeed, inhibition of MAO-B activity by selegiline was associated with attenuation of oxidative stress in models of neurological or cardiac disorders $(11,12)$, while its effect on CS-induced lung injury still needs to be investigated. We hypothesized that selegiline may play protective roles in CS-induced oxidative stress and inflammation in rat lungs in vivo. Oxidative stress-induced inflammation has been reported in previous studies $(13,14)$. Among the many proinflammatory cytokines, monocyte chemoattractant protein 1 (MCP-1) is one of the key chemokines that regulates macrophages infiltration and migration into inflamed sites (15). Macrophages and neutrophils are phagocytes that play major roles in the onset and maintenance of an inflammatory process. Neutrophils are the first immune cells to be recruited to the sites of injury, which can be through the cytokines produced by macrophages (16). During acute inflammation, neutrophils were first recruited by cytokine-induced neutrophil chemoattractant (CINC-1) to the inflamed sites, where they played an important role in host defense. Activated neutrophils were able to release myeloperoxidase (MPO) into extracellular milieu, where they used $\mathrm{H}_{2} \mathrm{O}_{2}$ to produce reactive hypochlorous acid, probably the most potent oxidant generated in vivo (17).

Interestingly, MAO-B inhibition by oral selegiline showed to be an effective smoking cessation medication in two early small size clinical studies $(18,19)$. In contrast, two other clinical studies indicated that oral or transdermal selegiline administration was not an effective aid for smoking cessation $(20,21)$. In order to support the protective effect of selegiline from in vitro findings (10), we first established an acute CS-exposed rat model using the SCIREQ inExpose whole body exposure system (SCIREQ, Montreal, Canada). We then evaluated the role of MAO-B inhibition by selegiline and elucidated the underlying mechanisms of selegiline on oxidative/inflammatory responses in rat lung and bronchoalveolar lavage (BAL).

We present the following article in accordance with the ARRIVE reporting checklist (available at: http://dx.doi. org/10.21037/atm-20-2426).

\section{Methods}

\section{Cigarette smoke-exposed rat model}

Thirty-two male Sprague-Dawley (S-D) rats (5-6 weeks old, 140-150 g) were purchased and housed in Laboratory Animal Unit (LAU) of The University of Hong Kong. The S-D rat strain, which is widely used in a variety of models, has been reported to be susceptible to CS-induced lung injury, oxidative stress and inflammation as end points $(22,23)$. It has appeared that age at exposure is an important stipulation for consideration of long-germ effects of smoking such as the development of COPD. It is well known that young children are much more susceptible to the effects of exposure to environmental tobacco smoke (ETS) compared to adults. ETS-induced early lung damage has been found in healthy male adolescents (24). This is why we have performed experiments on 5-6 weeks 
old rats. As our young rat model might provide a means of studying the mechanisms that control normal lung development and strategies for prevention of COPD in adult life. Cigarettes (10 mg tar and $0.8 \mathrm{mg}$ nicotine, Camel, R.J. Reynolds, Winston-Salem, NC, USA) were obtained from local commercial retailers. The rats were randomly divided into four groups ( $\mathrm{n}=8$ in each group): saline plus sham air (Saline/air), saline plus cigarette smoke (Saline/ $\mathrm{CS}$ ), selegiline plus sham air (Slg/air) and selegiline plus cigarette smoke (Slg/CS). Rats from Saline/air and Saline/ CS groups were intraperitoneally injected with saline $(2 \mathrm{~mL} /$ $\mathrm{kg}$ body weight) while rats from Slg/air and $\mathrm{Slg} / \mathrm{CS}$ groups were intraperitoneally injected with selegiline [dissolved in saline; $2 \mathrm{mg} / \mathrm{kg}$ body weight; Tocris (Missouri, UK)] about 30 min prior to CS exposure daily. The dose of selegiline was selected based upon one previous report (25), and the calculation from a simple practice guide for dose conversion between rat and human (26). Rats from Saline/air and Slg/ air groups were exposed to atmospheric air while rats from Saline/CS and Slg/CS groups were exposed to mainstream CS generated from the computer-controlled whole body inExpose smoking system (SCIREQ, Montreal, Canada) at a total particulate matter (TPM) of $2,000 \mathrm{mg} / \mathrm{m}^{3}$ for 1 hour (20 cigarettes) twice daily (am and $\mathrm{pm}$ ) for 7 consecutive days. The level of TPM was equivalent to our homemade smoking exposure system at 4\% CS in our previous publication (27). Rats were sacrificed by intraperitoneally injected with pentobarbitone $(100 \mathrm{mg} / \mathrm{kg}$ body weight) 24 hours after the last exposure. All animal procedures were performed in strict accordance with the guidelines from ARRIVE and Directive 2010/63/EU of the European Parliament, and the animal protocols were approved by the Committee on the Use of Live Animals in Teaching and Research (CULATR) of The University of Hong Kong (No. 3875-16).

\section{Cytological analysis of bronchoalveolar lavage (BAL)}

BAL from 2 lavages of each rat was pooled and counted by dual fluorescent acridine orange/ethidium bromide (AO/EB) assay for total cell count. A number of 40,000 cells from each sample were then centrifuged onto glass slides at $350 \mathrm{rpm}$ for 10 minutes by a cytospin device (CYTOPRO 7620, Wescor, UT, USA) for cytological analysis. After air dry overnight, the slides were stained with Differential Quick Stain Kit [(Modified Giemsa), Electron Microscopy Sciences, Hatfield, PA, USA] according to the manufacturer's instruction. Differential counts of white blood cells (neutrophils, macrophages and lymphocytes) were carried out under a light microscope (Nikon, Tokyo, Japan) and corrected for total cell count in BAL.

BAL was then centrifuged at $1,000 \times g$ for 10 minutes and the cell-free supernatants were frozen in aliquots for further assays.

\section{Protein extraction}

A small piece of frozen lung tissues (weighing 20-40 mg) from each rat were ground up to powder with a mortar and pestle in liquid nitrogen. To extract total protein (i.e., lung homogenates), powder was lysed in T-PER tissue protein extraction reagent (PIERCE, Rockford, IL, USA) in the presence of Halt ${ }^{\mathrm{TM}}$ protease and phosphatase inhibitor cocktail (Thermo Scientific, Rockford, IL, USA). Nuclear fraction was extracted by using the NE-PER Nuclear and Cytoplasmic Extraction Reagents (Thermo Scientific, Waltham, MA, USA) while mitochondrial fraction was extracted by using ProteoExtract Cytosol/ Mitochondria Fractionation Kit (Calbiochem) according to the manufacturers' instructions. Protein concentration was determined by Bradford protein assay (Bio-Rad Laboratories, Hercules, CA, USA). All protein samples were stored in aliquots at $-70{ }^{\circ} \mathrm{C}$ until further use.

\section{Determination of MAO-B activity}

MAO-B activity was measured by Monoamine Oxidase Activity Fluorometric Assay Kit (BioVision Incorporated, Milpitas, CA, US) according to the manufacturer's instruction. The MAO-B activity was corrected with lung protein concentration.

\section{Measurement of ROS levels in cytoplasmic and mitochondrial fraction}

Freshly prepared cytoplasmic fraction was incubated with $5 \mu$ M DCF-DA (Thermo Scientific) while the mitochondrial fraction was incubated with $5 \mu \mathrm{M}$ MitoSOX Red (Thermo Scientific) for 30 minutes at $37^{\circ} \mathrm{C}$ in dark. The fluorescent signal was determined using a fluorescence plate reader at appropriate wavelength (DCF: Ex/Em $=488 / 525 \mathrm{~nm}$; MitoSOX: $\mathrm{Ex} / \mathrm{Em}=510 / 580 \mathrm{~nm}$ ) for the measurement of ROS levels in the cytoplasmic and mitochondrial fraction.

\section{Measurement of oxidative stress markers}

The measurement of total antioxidant capacity (TAOC), 
superoxide dimutase (SOD) and catalase (CAT) activities, and GSH/GSSG ratio in lung homogenates were using commercially available kits. (Nanjing Jiancheng Bioengineering Institute, Nanjing, China). Results were normalized to lung protein concentration.

\section{ELISA for cytokines in bronchoalveolar lavage (BAL)}

Cytokines (CINC-1, R \& D Systems, Minneapolis, MN, USA; MCP-1, IL-6 and IL-10, BD Biosciences, San Jose, CA, USA) in BAL were measured by commercially available ELISA kits according to the manufacturers' instructions.

\section{Western blot analysis}

Samples containing equal amounts of protein were separated in $10 \%$ SDS-PAGE and transferred onto a nitrocellulose membrane. After blocking with $5 \%$ nonfat milk in tris-buffered saline ( $\mathrm{pH} 7.4$ ) containing $0.1 \%$ tween-20 (TBST), target proteins were detected using specified antibodies for analysis of HO-1 (ab13248, 1:1000, Abcam, Cambridge, UK), NQO1 (sc-32793, 1:200, Santa Cruz Biotechnology, Dallas, TX, USA), Nrf2 (sc-722, 1:200, Santa Cruz), NF-кB p65 (4764, 1:1000, Cell Signaling Technology, Danvers, MA, USA), pERK (4370, 1:1000, Cell Signaling Technology), ERK (sc-94, 1:2000, Santa Cruz Biotechnology), p-p38 (9211, 1:1000, Cell Signaling Technology), p38 (9212, 1:1000, Cell Signaling Technology), $\beta$-actin (\#4970, 1:2000, Santa Cruz Biotechnology), Lamin A/C (sc-20681, 1:2000, Santa Cruz Biotechnology). Then, the membranes were washed with TBST and later incubated with horseradish peroxidase (HRP)-conjugated secondary antibodies for 2 hours at room temperature. Afterward, the membranes were incubated with enhanced chemiluminescence (ECL) (GE Healthcare, Germany) and exposed to medical X-ray film (Fumingwei, Shenzhen, China). Densitometric analysis of the bands was performed with GelQuant (BiochemLabSolutions, UCSF, CA, US). Protein expression levels were normalized to $\beta$-actin for total/cytoplasmic protein and Lamin A/C for nuclear protein.

\section{Data and statistical analysis}

Numerical data were expressed as mean \pm SEM from 6-8 rats in each group. Statistical analyses were performed by one-way analysis of variance (ANOVA) followed by Tukey's post hoc test for comparison among multiple groups, or
Student's $t$ test for comparison between two groups where appropriate, using software Prism 7.0 (GraphPad). A value of $\mathrm{P}<0.05$ was regarded as statistically significant.

\section{Results}

\section{Selegiline inbibited CS-induced elevation of MAO-B activity}

Lung MAO-B activity was markedly elevated after CS exposure compared to Saline/SA group. As a selective $\mathrm{MAO}-\mathrm{B}$ inhibitor, selegiline alone significantly reduced MAO-B activity. Administration of selegiline before CS exposure reversed the CS-induced elevation of MAO-B activity significantly (Figure 1A). To further investigate the role of MAO-B in CS-induced oxidative stress, cytoplasmic and mitochondrial fractions from lung tissues were extracted to measure ROS levels. CS exposure showed no significant elevation of ROS levels in both cytoplasmic and mitochondrial fractions (Figure 1B,C).

\section{Selegiline reversed CS-induced alterations of oxidant/ antioxidant balance in lungs}

To reveal the overall anti-oxidative capacity, T-AOC was measured in rat lung homogenates. The levels of T-AOC were significantly decreased after CS exposure. Selegiline administration showed no significant reversal in CS-induced reduction of T-AOC (Figure $2 A$ ). CS exposure also caused significant reduction in rGSH/GSSG ratio (Figure $2 B$ ), significant increase in SOD activity (Figure 2C) but not in CAT activity (Figure 2D), indicating the presence of increased oxidative stress. Selegiline significantly reversed CS-induced reduction of rGSH/GSSG ratio, but not elevation of SOD activity. There was a significant higher rGSH/GSSG ratio in Slg/CS group compared to Saline/ air group (Figure $2 B$ ), suggesting that selegiline might have a direct protective effect on CS-induced oxidative response through modulation of endogenous antioxidant GSH level.

\section{Effect of selegiline on CS-induced HO-1, NQO1 protein expression and the involvement of Nrf2 signaling pathway}

CS exposure led to significant upregulation of HO-1 and NQO1 protein expression compared to Saline/ air group, which were significantly attenuated in the presence of selegiline in Slg/CS group. Selegiline alone had no effect on HO-1, but induced significant elevation 

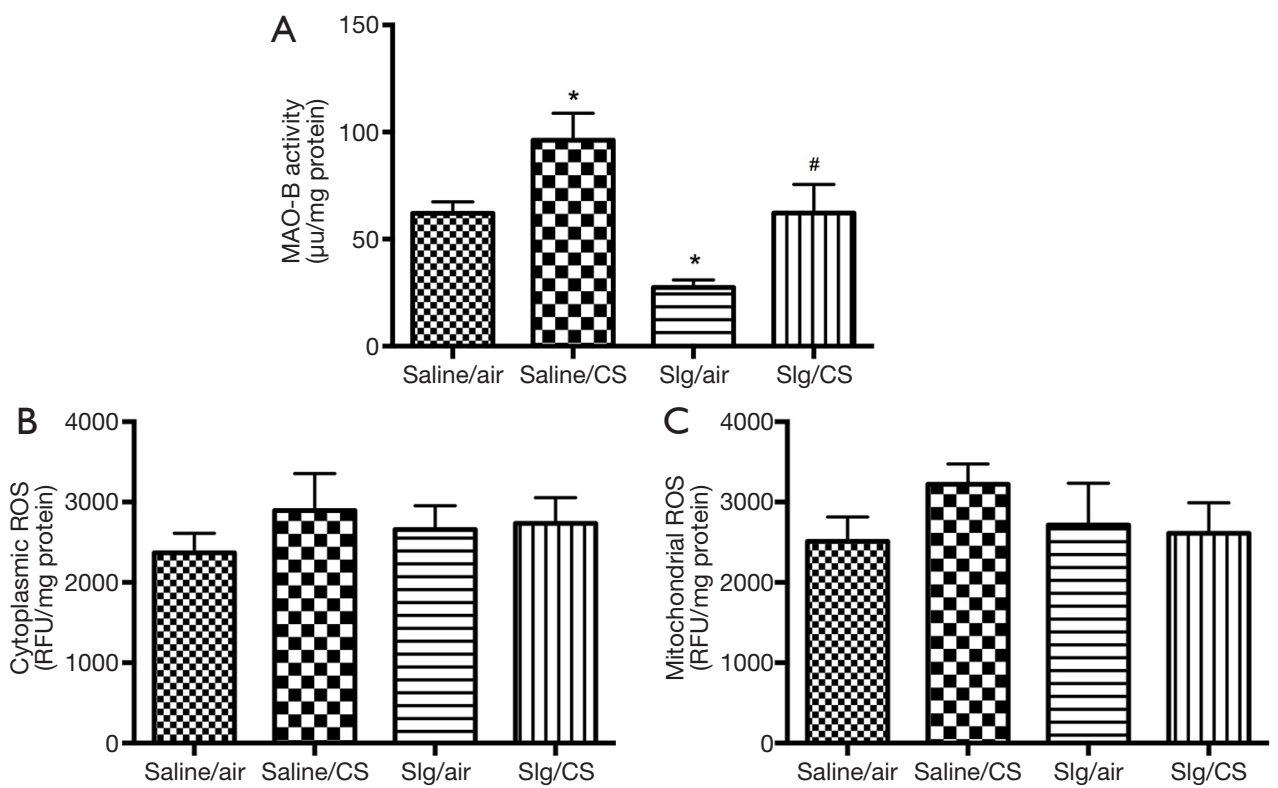

Figure 1 Effect of selegiline on MAO-B activity and ROS levels in rat lungs. Protein from rat lungs was extracted appropriately according to the protocols for the measurement of (A) MAO-B activity, (B) cytoplasmic ROS and (C) mitochondrial ROS levels. Results were corrected for protein concentration. Values are expressed as mean $\pm \mathrm{SEM}$ ( $\mathrm{n}=6-8)$. *, $\mathrm{P}<0.05$, compared with Saline/air group; ${ }^{\#}$, $\mathrm{P}<0.05$, compared with Saline/CS group (one-way ANOVA followed by Tukey's post hoc test).
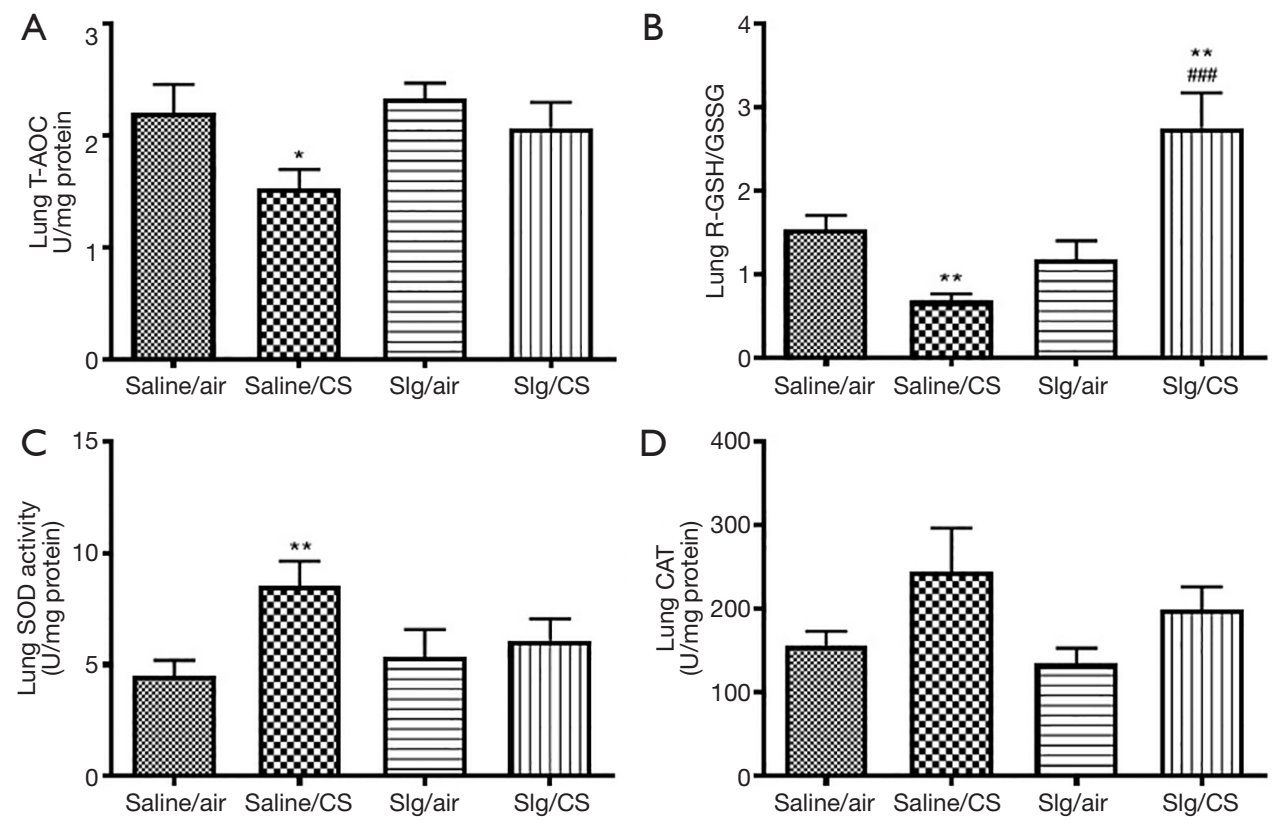

Figure 2 Effect of selegiline on CS-induced oxidant/antioxidant balance in rat lungs. Oxidant and anti-oxidant markers were measured using commercially available kits. Levels of (A) T-AOC, (B) GSH/GSSG ratio, (C) SOD activity and (D) CAT activity were measured in rat lungs. Results were corrected with protein concentration and expressed as mean \pm SEM ( $\mathrm{n}=7-8)$. *, $\mathrm{P}<0.05$; **, $\mathrm{P}<0.01$, compared with Saline/air group; ${ }^{\# \#}, \mathrm{P}<0.001$, compared with Saline/CS group (one-way ANOVA followed by Tukey's post hoc test). 
A

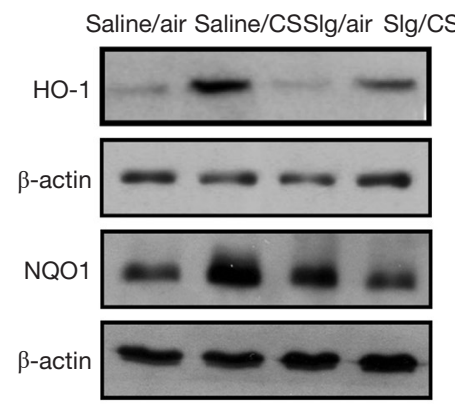

W Saline/air

Daline/CS

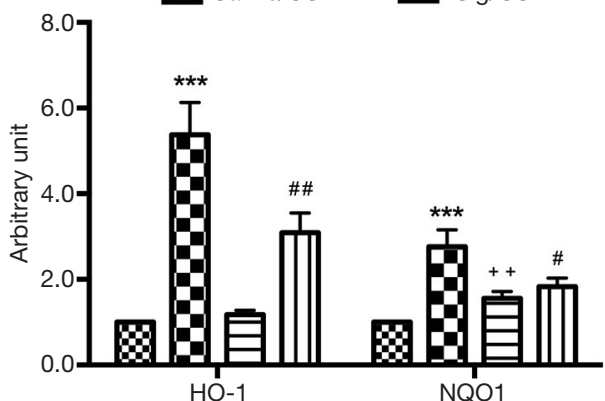

B Saline/air Saline/CSSlg/air Slg/CS

Nrf2 cytoplasmic

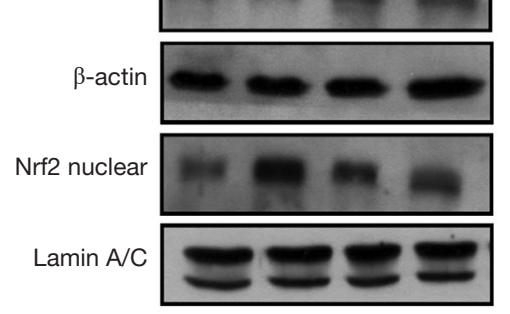

$\boldsymbol{B}$ Saline/air $\mathbf{E S I g / a i r}$

ㅁ] Saline/CS

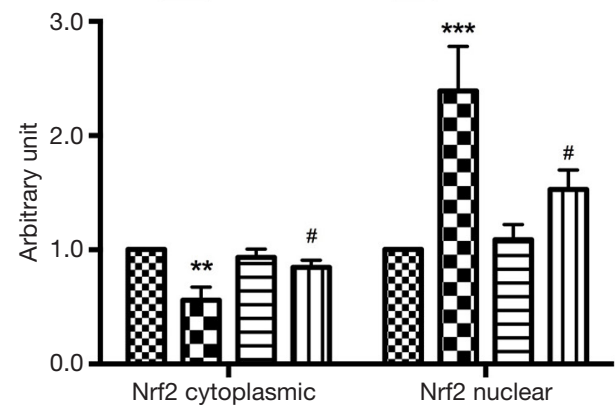

Figure 3 Effect of selegiline on CS-induced HO-1 and NQO1 protein expression and Nrf2 nuclear translocation in rat lungs. Total protein and cytoplasmic and nuclear protein from rat lungs were extracted for Westerm blots. (A) Representative Western blots and quantification of the effect of selegiline on CS-induced HO-1 and NQO1 protein expression. Total protein was normalized to $\beta$-actin. (B) Representative Western blots and quantification of the effect of selegiline on CS-induced Nrf2 nuclear translocation. Cytoplasmic protein was normalized to $\beta$-actin while nuclear protein was normalized to Lamin $\mathrm{A} / \mathrm{C}$. Values are expressed as mean $\pm \mathrm{SEM}(\mathrm{n}=8)$. ${ }^{* *}, \mathrm{P}<0.01 ;{ }^{* * *}, \mathrm{P}<0.001$,

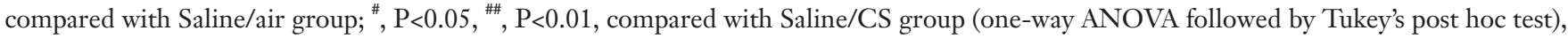
${ }^{++}, \mathrm{P}<0.01$ compared with Saline/air group (unpaired Student's $t$ test).

of NQO1 protein expression (Figure $3 A$ ). As Nrf2 is the key regulator of cytoprotective genes in the adaptive response to oxidative stress, we next investigated the effect of selegiline in this pathway under CS exposure. Translocation of Nrf2 from cytoplasmic fractions to nuclear fractions was observed in Saline/CS group. Selegiline alone had no effect, but blocked the CS-induced $\mathrm{Nrf} 2$ translocation to the nucleus (Figure 3B).

\section{Effect of selegiline on CS-induced infiltration of inflammatory cells in BAL}

Total cell counts in BAL were measured by AO/EB assay as an indication of infiltration of inflammatory cells into the lungs. Diff-Quick staining was performed on cytospinned BAL cells. The Saline/CS group demonstrated significant elevation of total cell counts, especially the number of macrophages but not the number of neutrophils or lymphocytes in BAL compared to Saline/air group (Figure 4) due to large variability among each rat within groups. Selegiline alone or in combination with CS (Slg/ CS group) had no significant effects on total and individual inflammatory cell counts in BAL.

\section{Selegiline reversed CS-induced elevation of pro- inflammatory mediators and restored CS-induced reduction of anti-inflammatory mediator in $B A L$}

To further investigate the effect of selegiline on CSinduced inflammatory responses, levels of pro-inflammatory mediators including CINC-1, MCP-1, IL-6 and antiinflammatory mediator IL-10 were measured in BAL. Levels of CINC-1, MCP-1 and IL-6 in BAL were significantly elevated in Saline/CS group compared to Saline/air group, which was abated by selegiline (Figure $5 A, B, C)$. On the other hand, a significant reduction 

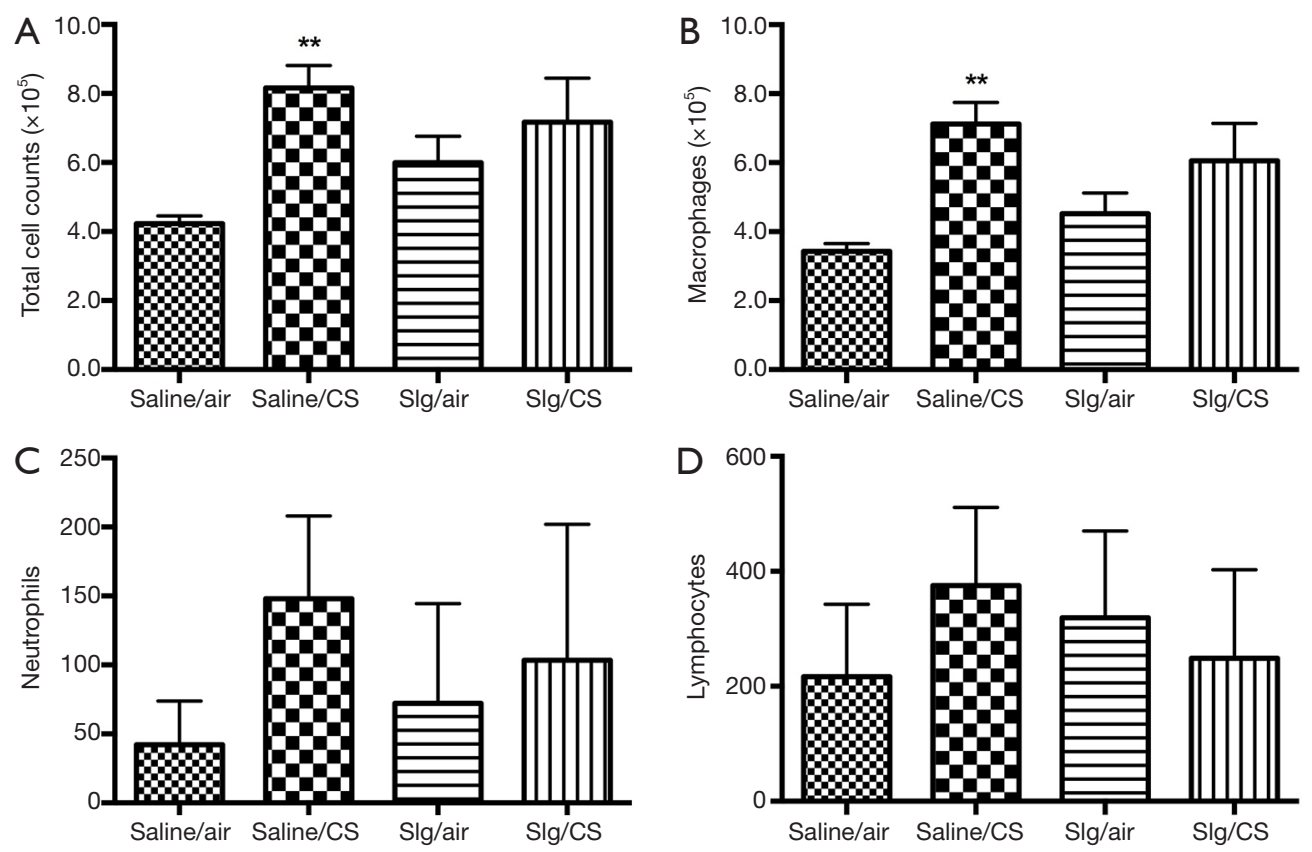

Figure 4 Effect of selegiline on total and differential cell counts in BAL after CS exposure. Total cells counts were measured by dual AO/EB staining. BAL was subjected to cytospin and stained by Diff-Quick staining Kit. Macrophages, neutrophils and lymphocytes were identified based on their color and morphology, and counted. (A) Total cell counts, (B) macrophages, (C) neutrophils and (D) lymphocytes in BAL were shown. Values are expressed as mean \pm SEM $(n=6)$. **, $\mathrm{P}<0.01$, compared with Saline/air group (one-way ANOVA followed by Tukey's post hoc test).
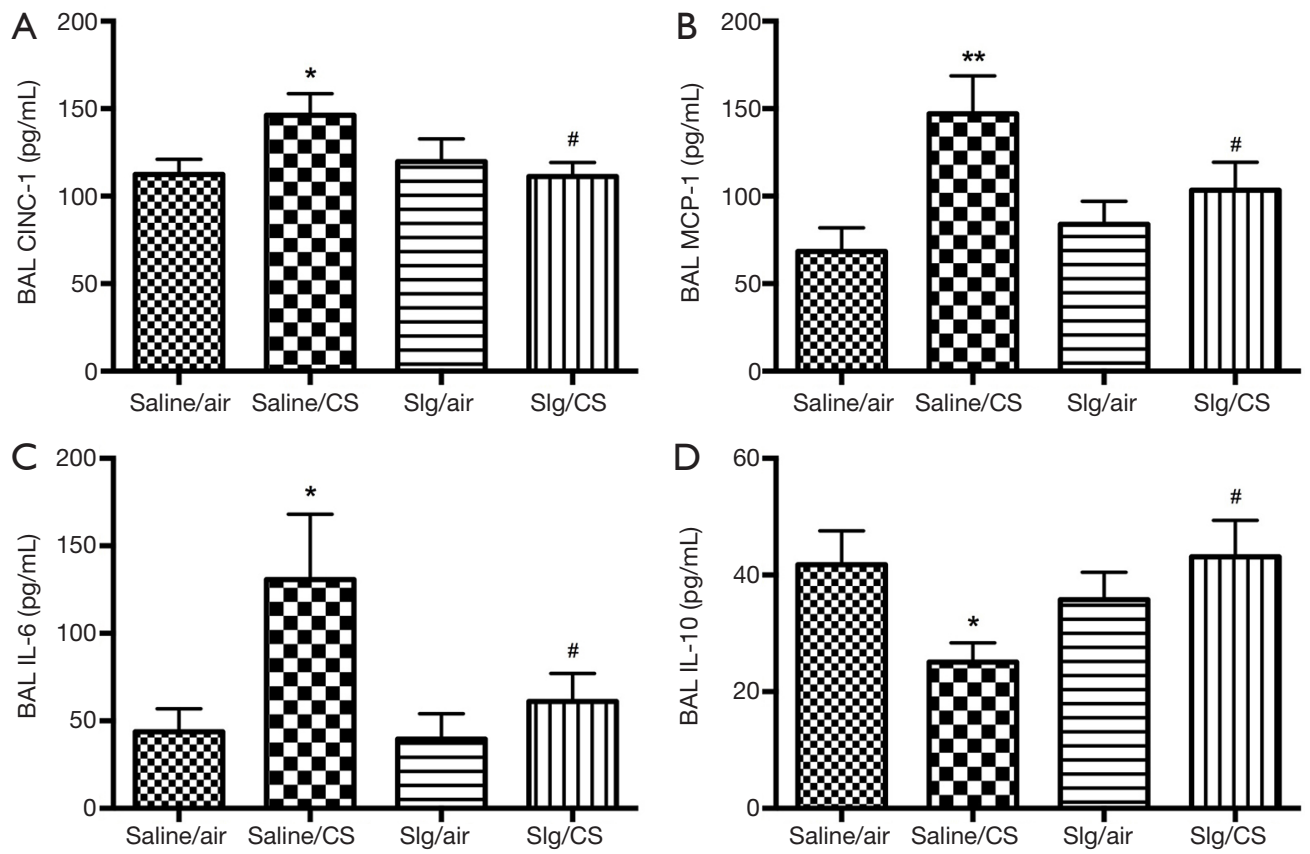

Figure 5 Effect of selegiline on CS-induced inflammatory responses in BAL. Levels of (A) CINC-1, (B) MCP-1, (C) IL-6, (D) IL-10 in BAL were measured using ELISA kits. Values are expressed as mean $\pm \mathrm{SEM}(\mathrm{n}=8)$. *, $\mathrm{P}<0.05$; ** $\mathrm{P}<0.01$, compared with $\mathrm{SA}$ control; \#, $\mathrm{P}<0.05$, compared with CS group (one-way ANOVA followed by Tukey's post hoc test). 

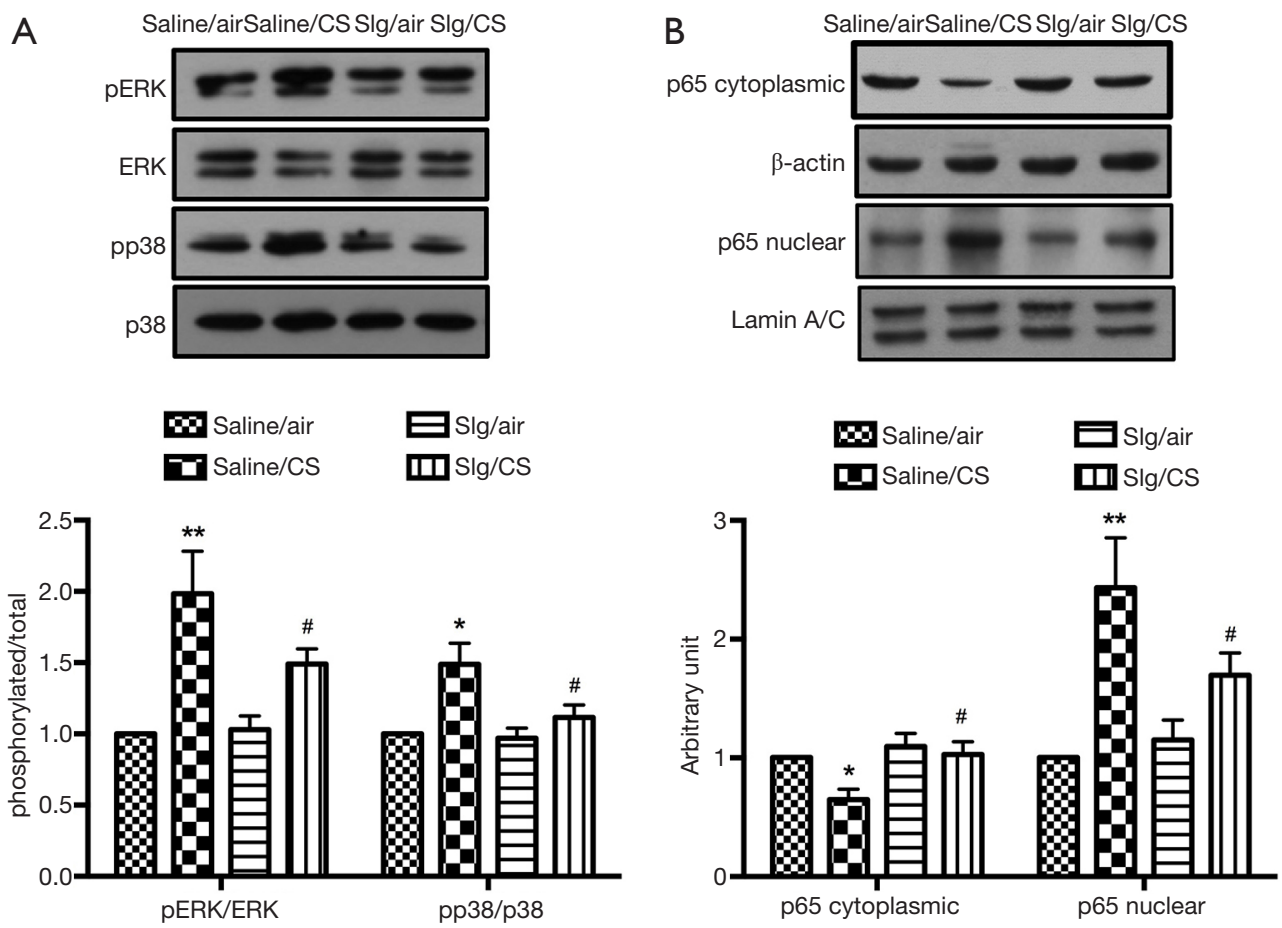

Figure 6 Effect of selegiline on CS-induced activation of MAPK and NF- $\kappa$ B signaling pathways in rat lungs. Total protein from rat lungs was extracted for the measurement of ERK and p38. (A) Representative Western blots and quantification of the effect of selegiline on CSinduced phosphorylation of ERK and p38. Phosphorylation of ERK was normalized to total ERK; phosphorylation of p38 was normalized to total p38. Nuclear and cytoplasmic fractions from rat lungs were extracted for the measurement of NF- $\mathrm{B}$ p65. (B) Representative Western blots and quantification of the effect of selegiline on CS-induced NF- $\kappa \mathrm{B}$ p65 protein expression in cytoplasmic and nuclear fractions. Cytoplasmic protein was normalized to $\beta$-actin; nuclear protein expression was normalized to Lamin A/C. Values are expressed as mean $\pm \operatorname{SEM}(\mathrm{n}=7-8) .{ }^{*}, \mathrm{P}<0.05 ;{ }^{* *}, \mathrm{P}<0.01$, compared with Saline/air group; ${ }^{*}, \mathrm{P}<0.05$, compared with Saline/CS group (one-way ANOVA followed by Tukey's post hoc test).

of anti-inflammatory mediator IL-10 levels was observed in BAL of Saline/CS group compared to Saline/air group, which was reversed by selegiline (Figure 5D). Selegiline alone had no effect on pro- and anti-inflammatory mediators.

\section{The involvement of NF- $\mathrm{KB}$ and MAPK signaling pathways in CS-induced inflammation}

To further investigate the underlying mechanism of inflammation, accumulated evidence have demonstrated that CS-derived oxidants can trigger several signaling pathways to regulate CS-induced inflammatory response, including MAPK and NF- $\kappa \mathrm{B}$ signaling pathways (28), which was being examined. Increased phosphorylation of ERK1/2 and $\mathrm{p} 38$ was observed after CS exposure in Saline/CS group compared to Saline/air group, which was attenuated by selegiline (Figure $6 A$ ). Protein expression of NF- $\mathrm{BB}$ p 65 was significantly reduced in cytoplasmic fractions and elevated in nuclear fractions of Saline/CS group compared to Saline/ air group. Pretreatment with selegiline suppressed CSinduced nuclear translocation of NF-kB p65 (Figure $6 B$ ).

\section{Discussion}

In this study, we demonstrated that the protective effect of selegiline on CS-induced lung oxidative stress and inflammation might be mainly through its inhibition on MAO-B, leading to the impairment of ROS production and inflammatory response via Nrf2 and NF- $\mathrm{KB}$ pathways. To the best of our knowledge, this is the first in vivo study of selegiline on the endogenous oxidative and redox system in lung. Oxidative stress-associated airway inflammation is believed to be the key components in the pathogenesis 
of COPD (29). Therefore, a better understanding of the underlying mechanisms of CS-induced oxidative stress is vital for the treatment of COPD. This study was in support of our previous in vitro study, which demonstrated that MAO-B was one of the ROS sources upon cigarette smoke medium (CSM) exposure in airway epithelial cells (10).

Cotinine is the primary metabolite of nicotine and is used as a biomarker for smokers due to its longer halflife compared to that of nicotine. In a pilot study of acute (7 days) CS exposure using the SCIREQ inExpose whole body exposure system with a concentration of $2,000 \mathrm{mg} / \mathrm{m}^{3}$ TPM of air, we demonstrated that serum cotinine levels were significantly elevated in Saline/CS group compared to Saline/air group (data not shown), suggesting the reliability of the smoking system for the establishment of passive smoking CS-exposed rat model.

In consistent with the in vitro findings by our group (10), a significant increase of MAO-B activity was observed in rat lungs after CS exposure in vivo. However, CS exposure showed no significant elevation of ROS levels in cytoplasmic and mitochondrial fractions, which might be due to the limitations of DCF-DA and MitoSOX including instability of the probes and their products (30). As CS-induced oxidative stress might be partially MAO$\mathrm{B}$-dependent, MAO-B could be a potential target for eliminating CS-induced oxidative stress in the lungs. To further confirm the involvement of MAO-B in oxidative stress, a selective MAO-B inhibitor selegiline was used to test this hypothesis. Selegiline is a useful drug in adjuvant therapy for Parkinson's disease. Besides its MAO-B inhibition, the antioxidant activity has also been demonstrated in animal models of brain injury and heart failure $(11,31,32)$ but less well known in the lungs. In this study, selegiline significantly reduced the CS-induced elevation of lung MAO-B activity, but showed no effect on cytoplasmic and mitochondrial ROS levels, which might be due to limitation of the assays using fluorescent probes (33). Therefore we further determined the antioxidant activity of selegiline in the lungs. Following CS exposure, the endogenous antioxidant defense system plays an important role in the regulation of oxidant/antioxidant balance. CS exposure caused significant reduction in T-AOC and GSH/GSSG ratio, demonstrating the defective antioxidative defense system in rat lungs. Despite of CSinduced significant elevation of SOD activity but not CAT activity, this indicated an effort of antioxidant defense mechanism to normalize CS-induced oxidative stress, in agreement with our previous report (27). However, inhibition of MAO-B by selegiline did not significantly restore CS-induced reduction of T-AOC and upregulation of SOD activity but significantly prevented CS-induced depletion of rGSH. In the current study, CS exposure initiated an adaptive response through activation of $\mathrm{Nrf} 2$ signaling pathway in combating oxidative stress, leading to dramatic induction of $\mathrm{Nrf} 2$ target genes including HO-1 and NQO1 in rat lungs, which was reversed by selegiline, in agreement with the in vitro findings (10). Under homeostatic conditions, unactivated Nrf2 is present in the cytoplasm attaching to a cytosolic protein Keap1, which functions as a suppressor of Nrf2 by retaining it in cytosol and enhancing its proteasomal degradation via ubiquitination (34). Nrf2 was released from Keap1/Nrf2 complex and translocate to the nucleus under oxidative stress (35). In this study, selegiline blocked CS-induced Nrf2 nuclear translocation.

The lung is a vital organ for gaseous exchange thus it is frequently exposed to environmental insulting agents in the air (36). In the lung defense system, the epithelium is the first site to be exposed to the inhaled insults (37). Epithelial cells shield the respiratory tract via secreting a variety of substances including lysozymes, mucins, lactoferrins and defensins (36). Following insult, epithelial cells and macrophages in the respiratory tract were capable of producing cytokines and chemokines to recruit more inflammatory cells, including macrophages, neutrophils and lymphocytes to the lungs, where they played a crucial role in host protection through phagocytosis and promoting local inflammation $(36,38)$. It has been demonstrated that macrophages were the principal resident phagocytes in the lungs (39-41). In agreement with these literatures, our results from Diff-Quick staining demonstrated that $>80 \%$ of total cell counts were macrophages in BAL. Oxidative stress-induced inflammation has been reported in previous studies $(13,14)$. Among the many pro-inflammatory cytokines, MCP-1 is one of the key chemokines that regulate macrophages infiltration and migration into inflamed sites (15). CS exposure caused an elevation of MCP-1 levels in BAL, which was suppressed by selegiline. On the other hand, neutrophils are the first immune cells to be recruited to the sites of injury (16). During acute inflammation, neutrophils were first recruited by CINC-1 to the inflamed sites, where they played an important role in host defense. CINC-1 levels in BAL were also increased after CS exposure, which was attenuated by selegiline.

Inflammation was necessary to fight off the harmful 


\section{Page 10 of 13}

insults; however excessive pro-inflammatory responses can lead to uncontrolled lung damage (36). Thus effective down-regulation of the inflammatory responses was important to maintain homeostasis. Anti-inflammatory cytokine like IL-10 was quite important to down-regulate the inflammatory responses. In this study, there was a significant up-regulation of pro-inflammatory mediators like CINC-1, MCP-1 and IL-6, and reduction of IL-10 in the Saline/CS group, leading to a shift to pro-inflammatory responses. Selegiline prevented CS-induced elevation of pro-inflammatory cytokines and reduction of antiinflammatory cytokine as evident by a tendency of reduction of cell counts in BAL.

Mitogen-activated protein kinase (MAPK, including ERK, p38, and JNK), are one of the best-known signaling pathways in the regulation of lung inflammation, and could be activated in a cell-stimulus-specific manner (42). The activation of MAPK signaling pathway including ERK and p38 has been reported in COPD patients or in cigarette smoke extract-exposed BEAS-2B cells $(43,44)$, leading to the induction of pro-inflammatory mediators. Therefore, attenuation of pro-inflammatory mediators release by selegiline could be due to the inactivation of ERK and p38 in MAPK signaling pathway. The NF- $\kappa \mathrm{B}$ signaling pathway was downstream of MAPK signaling pathway and could be activated by various types of stimuli such as oxidative stress and cytokines (45). Moreover, it has been demonstrated that CS was able to activate $\mathrm{NF}-\kappa \mathrm{B}$, a transcription factor associated with the production of inflammatory cytokines such as IL-6 and IL-8 in many cell types, such as alveolar epithelial cells, macrophages and bronchial epithelial cells (46-48). Activation of NF- $\mathrm{KB}$ signaling pathway was linked to inflammation in COPD (49). In this study, CS-induced $\mathrm{NF}-\kappa \mathrm{B}$ activation was suppressed by selegiline, which might be attributed to the impairment of ROS production via MAO-B inhibition.

There were limitations in the current study. First, the use of acute CS-exposed rats instead of long-term CS-exposed rats could not mimic emphysema, the key feature of COPD, which might be developed as a result of prolonged smokinginduced oxidative stress and inflammation. Previous findings observed that short term CS exposure (even less than 7 days) was able to produce oxidative stress and inflammation, clinical features of COPD, in Sprague-Dawley rats (50). Second, we did not clarify the mechanism governing the inactivation of NF- $\mathrm{\kappa B}$ by selegiline in the acute CS-exposed rats. The possibility of MAO-B-independent response might be present. Further studies using MAO-B knockout mice will be needed to clarify MAO-B-dependent or MAO$\mathrm{B}$-independent mechanisms of selegiline. Xiao and coworkers reported that MAO-B knockdown reduced the accumulation of 1-methyl-4-phenylpyridinium $\left(\mathrm{MPP}^{+}\right)$induced ROS in PC12 cells, and selegiline pretreatment preserved the antioxidative activity in MAO-B-knockdown PC cells (51). In addition to its blockade of MAO-B, selegiline also possesses a novel antioxidant effect through suppression of hydroxy radical formation (52). Third, data from human study indicated that MAO-B activities in the lungs of smokers were reduced rather than elevated in comparison to normal subjects $(53,54)$, which was in contradictory to our current findings. The possible reason for such a discrepancy could be that the smokers who were included would probably have been smoking for a long period of time, and hence the body's capabilities in synthesizing and/or turning over of MAO-B might have been greatly reduced. In fact, it has been suggested that it was necessary for a smoker to have been chronically exposed to CS in order to observe a drop in MAO-B level. These limitations need to be addressed in future studies.

Loss of MAO-B in human is associated with mental disorder such as depression, in which selegiline is an antidepressant used as a skin-patch. According to a study by Norwood and Balkissoon in 2005, the prevalence of depression in COPD patients was $40 \%$ compared to $15 \%$ in general population (55). Therefore, selegiline could reduce ROS and inflammatory response in the lung of COPD patients and at the same time could reduce depression through its role in the brain. Furthermore, a nationwide retrospective cohort study demonstrated that PD risk of Parkinson's disease was significantly increased in patients with COPD compared with those of the general population (56). Although there was currently insufficient evidence to support the efficacy of selegiline for the treatment of COPD, a monotherapeutic agent to treat both conditions (either COPD/depression or COPD/Parkinson's disease) would be a valuable therapeutic strategy. However, it remains uncertain what the effective dose of selegiline should be to treat COPD/depression or COPD/Parkinson's disease.

In conclusion, our findings suggest that MAO-B inhibitor selegiline may be capable of reversing CS-induced oxidative stress and inflammation via $\mathrm{Nrf} 2$ and $\mathrm{NF}-\kappa \mathrm{B}$ signaling pathways (Figure 7). Inhibition of MAO-B may provide a promising therapeutic strategy for CS-mediated oxidative stress and inflammation in acute CS-exposed rat lungs. 


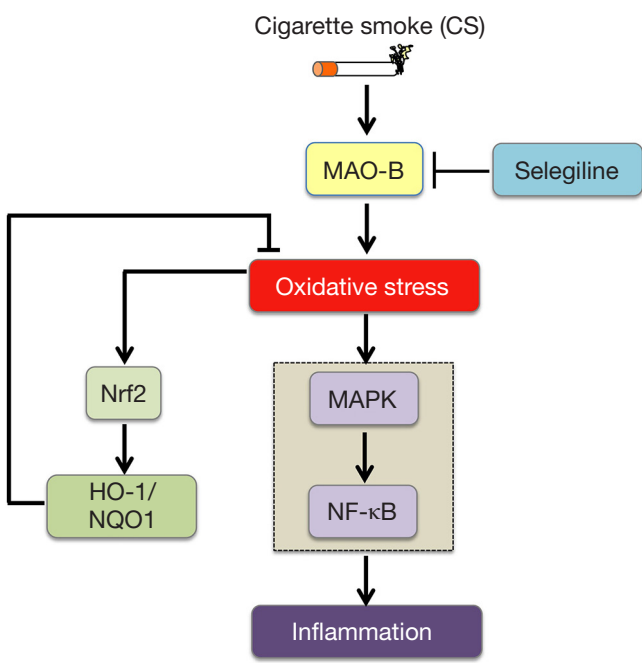

Figure 7 A schematic diagram to demonstrate the effect of selegiline on CS-induced oxidative stress and inflammation. MAO-B is an important ROS source located at the outer membrane of mitochondria. After CS exposure, MAO-B activity was increased thus contributing to CS-induced oxidative stress and inflammation via activation of $\mathrm{Nrf} 2$ and $\mathrm{MAPK} / \mathrm{NF}-\kappa \mathrm{B}$ signaling pathways. The selective MAO-B inhibitor selegiline partially blocked CS-induced oxidative stress and inflammation through inhibition of MAO-B-derived ROS.

\section{Acknowledgments}

Funding: This study was supported by Stanley Ho Matching Grant (208040027) and YC Chan Scientist Award (200007691) from The University of Hong Kong.

\section{Footnote}

Reporting Checklist: The authors present the study in accordance with the ARRIVE Reporting Checklist. Available at http://dx.doi.org/10.21037/atm-20-2426

Data Sharing Statement: Available at http://dx.doi. org/10.21037/atm-20-2426

Peer Review File: Available at http://dx.doi.org/10.21037/ atm-20-2426

Conflicts of Interest: All authors have completed the ICMJE uniform disclosure form (available at http://dx.doi. org/10.21037/atm-20-2426). The authors have no conflicts of interest to declare.
Ethical Statement: The authors are accountable for all aspects of the work in ensuring that questions related to the accuracy or integrity of any part of the work are appropriately investigated and resolved. All animal procedures were performed in strict accordance with the guidelines from ARRIVE and Directive 2010/63/EU of the European Parliament, and the animal protocols were approved by the Committee on the Use of Live Animals in Teaching and Research (CULATR) of The University of Hong Kong (No. 3875-16).

Open Access Statement: This is an Open Access article distributed in accordance with the Creative Commons Attribution-NonCommercial-NoDerivs 4.0 International License (CC BY-NC-ND 4.0), which permits the noncommercial replication and distribution of the article with the strict proviso that no changes or edits are made and the original work is properly cited (including links to both the formal publication through the relevant DOI and the license). See: https://creativecommons.org/licenses/by-nc-nd/4.0/.

\section{References}

1. Vestbo J, Hurd SS, Agusti AG, et al. Global strategy for the diagnosis, management, and prevention of chronic obstructive pulmonary disease: GOLD executive summary. Am J Respir Crit Care Med 2013;187:347-65.

2. Lopez AD, Shibuya K, Rao C, et al. Chronic obstructive pulmonary disease: current burden and future projections. Eur Respir J 2006;27:397-412.

3. Lopez-Campos JL, Tan W, Soriano JB. Global burden of COPD. Respirology 2016;21:14-23.

4. Wiegman CH, Michaeloudes C, Haji G, et al. Oxidative stress-induced mitochondrial dysfunction drives inflammation and airway smooth muscle remodeling in patients with chronic obstructive pulmonary disease. J Allergy Clin Immunol 2015;136:769-80.

5. Sunil VR, Vayas KN, Massa CB, et al. Ozone-Induced Injury and Oxidative Stress in Bronchiolar Epithelium Are Associated with Altered Pulmonary Mechanics. Toxicol Sci 2013;133:309-19.

6. Holguin F. Oxidative stress in airway diseases. Ann Am Thorac Soc 2013;10 Suppl:S150-7.

7. Murphy MP. How mitochondria produce reactive oxygen species. Biochem J 2009;417:1-13.

8. Edmondson DE. Hydrogen peroxide produced by mitochondrial monoamine oxidase catalysis: biological implications. Curr Pharm Des 2014;20:155-60. 
9. Puente-Maestu L, Perez-Parra J, Godoy R, et al. Abnormal mitochondrial function in locomotor and respiratory muscles of COPD patients. Eur Respir J 2009;33:1045-52.

10. Cui Y, Liu KW, Liang Y, et al. Inhibition of monoamine oxidase-B by selegiline reduces cigarette smoke-induced oxidative stress and inflammation in airway epithelial cells. Toxicol Lett 2017;268:44-50.

11. Qin F, Shite J, Mao W, et al. Selegiline attenuates cardiac oxidative stress and apoptosis in heart failure: association with improvement of cardiac function. Eur J Pharmacol 2003;461:149-58.

12. Takahata K, Shimazu S, Katsuki H, et al. Effects of selegiline on antioxidant systems in the nigrostriatum in rat. J Neural Transm (Vienna) 2006;113:151-8.

13. Gloire G, Legrand-Poels S, Piette J. NF-kappaB activation by reactive oxygen species: fifteen years later. Biochem Pharmacol 2006;72:1493-505.

14. Son Y, Kim S, Chung HT, et al. Reactive oxygen species in the activation of MAP kinases. Methods Enzymol 2013;528:27-48.

15. Melgarejo E, Medina MA, Sanchez-Jimenez F, et al. Monocyte chemoattractant protein-1: a key mediator in inflammatory processes. Int J Biochem Cell Biol 2009;41:998-1001.

16. Abraham E. Neutrophils and acute lung injury. Crit Care Med 2003;31:S195-9.

17. Klebanoff SJ. Myeloperoxidase: friend and foe. J Leukoc Biol 2005;77:598-625.

18. George TP, Vessicchio JC, Termine A, et al. A preliminary placebo-controlled trial of selegiline hydrochloride for smoking cessation. Biol Psychiatry 2003;53:136-43.

19. Biberman R, Neumann R, Katzir I, et al. A randomized controlled trial of oral selegiline plus nicotine skin patch compared with placebo plus nicotine skin patch for smoking cessation. Addiction 2003;98:1403-7.

20. Weinberger AH, Reutenauer EL, Jatlow PI, et al. A double-blind, placebo-controlled, randomized clinical trial of oral selegiline hydrochloride for smoking cessation in nicotine-dependent cigarette smokers. Drug Alcohol Depend 2010;107:188-95.

21. Killen JD, Fortmann SP, Murphy GM, Jr., et al. Failure to improve cigarette smoking abstinence with transdermal selegiline + cognitive behavior therapy. Addiction 2010;105:1660-8.

22. Wang G, Mohammadtursun N, Sun J, et al. Establishment and Evaluation of a Rat Model of Sidestream Cigarette Smoke-Induced Chronic Obstructive Pulmonary Disease. Front Physiol 2018;9:58.
23. Ghorani V, Boskabady MH, Khazdair MR, et al. Experimental animal models for COPD: a methodological review. Tob Induc Dis 2017;15:25.

24. Rizzi M, Sergi M, Andreoli A, et al. Environmental tobacco smoke may induce early lung damage in healthy male adolescents. Chest 2004;125:1387-93.

25. Yohn SE, Reynolds S, Tripodi G, et al. The monoamineoxidase B inhibitor deprenyl increases selection of higheffort activity in rats tested on a progressive ratio/chow feeding choice procedure: Implications for treating motivational dysfunctions. Behav Brain Res 2018;342:27-34.

26. Nair AB, Jacob S. A simple practice guide for dose conversion between animals and human. J Basic Clin Pharm 2016;7:27-31.

27. Chan KH, Ho SP, Yeung SC, et al. Chinese green tea ameliorates lung injury in cigarette smoke-exposed rats. Respir Med 2009;103:1746-54.

28. Foronjy R, D'Armiento J. The Effect of Cigarette Smokederived Oxidants on the Inflammatory Response of the Lung. Clin Appl Immunol Rev 2006;6:53-72.

29. Decramer M, Janssens W, Miravitlles M. Chronic obstructive pulmonary disease. Lancet 2012;379:1341-51.

30. Dikalov SI, Harrison DG. Methods for detection of mitochondrial and cellular reactive oxygen species. Antioxid Redox Signal 2014;20:372-82.

31. Kitani K, Minami C, Isobe K, et al. Why (--)deprenyl prolongs survivals of experimental animals: increase of anti-oxidant enzymes in brain and other body tissues as well as mobilization of various humoral factors may lead to systemic anti-aging effects. Mech Ageing Dev 2002;123:1087-100.

32. Wu RM, Chiueh CC, Pert A, et al. Apparent antioxidant effect of 1-deprenyl on hydroxyl radical formation and nigral injury elicited by MPP+ in vivo. Eur J Pharmacol 1993;243:241-7.

33. Kalyanaraman B, Darley-Usmar V, Davies KJ, et al. Measuring reactive oxygen and nitrogen species with fluorescent probes: challenges and limitations. Free Radic Biol Med 2012;52:1-6.

34. Baird L, Dinkova-Kostova AT. The cytoprotective role of the Keap1-Nrf2 pathway. Arch Toxicol 2011;85:241-72.

35. Kensler TW, Wakabayashi N, Biswal S. Cell survival responses to environmental stresses via the Keap1Nrf2-ARE pathway. Annu Rev Pharmacol Toxicol 2007;47:89-116.

36. Moldoveanu B, Otmishi P, Jani P, et al. Inflammatory mechanisms in the lung. J Inflamm Res 2009;2:1-11.

37. Gao W, Li L, Wang Y, et al. Bronchial epithelial cells: The 
key effector cells in the pathogenesis of chronic obstructive pulmonary disease? Respirology 2015;20:722-9.

38. Smith LA, Paszkiewicz GM, Hutson AD, et al. Inflammatory response of lung macrophages and epithelial cells to tobacco smoke: a literature review of ex vivo investigations. Immunol Res 2010;46:94-126.

39. Laan M, Cui ZH, Hoshino H, et al. Neutrophil recruitment by human $\mathrm{IL}-17$ via $\mathrm{C}-\mathrm{X}-\mathrm{C}$ chemokine release in the airways. J Immunol 1999;162:2347-52.

40. Prasse A, Stahl M, Schulz G, et al. Essential role of osteopontin in smoking-related interstitial lung diseases. Am J Pathol 2009;174:1683-91.

41. Shore SA, Abraham JH, Schwartzman IN, et al. Ventilatory responses to ozone are reduced in immature rats. J Appl Physiol (1985) 2000;88:2023-30.

42. Raman $M$, Chen W, Cobb MH. Differential regulation and properties of MAPKs. Oncogene 2007;26:3100-12.

43. Renda T, Baraldo S, Pelaia G, et al. Increased activation of p38 MAPK in COPD. Eur Respir J 2008;31:62-9.

44. Li D, Hu J, Wang T, et al. Silymarin attenuates cigarette smoke extract-induced inflammation via simultaneous inhibition of autophagy and ERK/p38 MAPK pathway in human bronchial epithelial cells. Sci Rep 2016;6:37751.

45. Wright JG, Christman JW. The role of nuclear factor kappa $\mathrm{B}$ in the pathogenesis of pulmonary diseases: implications for therapy. Am J Respir Med 2003;2:211-9.

46. Moodie FM, Marwick JA, Anderson CS, et al. Oxidative stress and cigarette smoke alter chromatin remodeling but differentially regulate NF-kappaB activation and proinflammatory cytokine release in alveolar epithelial cells. FASEB J 2004;18:1897-9.

47. Yang SR, Chida AS, Bauter MR, et al. Cigarette smoke induces proinflammatory cytokine release by activation of NF-kappaB and posttranslational modifications of histone deacetylase in macrophages. Am J Physiol Lung Cell Mol
Physiol 2006;291:L46-57.

48. Syed DN, Afaq F, Kweon MH, et al. Green tea polyphenol EGCG suppresses cigarette smoke condensate-induced NF-kappaB activation in normal human bronchial epithelial cells. Oncogene 2007;26:673-82.

49. Di Stefano A, Caramori G, Oates T, et al. Increased expression of nuclear factor-kappaB in bronchial biopsies from smokers and patients with COPD. Eur Respir J 2002;20:556-63.

50. Stevenson CS, Docx C, Webster R, et al. Comprehensive gene expression profiling of rat lung reveals distinct acute and chronic responses to cigarette smoke inhalation. Am J Physiol Lung Cell Mol Physiol 2007;293:L1183-93.

51. Xiao H, Lv F, Xu W, et al. Deprenyl prevents MPP(+)induced oxidative damage in PC12 cells by the upregulation of Nrf2-mediated NQO1 expression through the activation of PI3K/Akt and Erk. Toxicology 2011;290:286-94.

52. Wu RM, Chen RC, Chiueh CC. Effect of MAO-B inhibitors on MPP+ toxicity in Vivo. Ann N Y Acad Sci 2000;899:255-61.

53. Fowler JS, Logan J, Wang GJ, et al. Low monoamine oxidase B in peripheral organs in smokers. Proc Natl Acad Sci U S A 2003;100:11600-5.

54. Berlin I, Said S, Spreux-Varoquaux O, et al. Monoamine oxidase A and B activities in heavy smokers. Biol Psychiatry 1995;38:756-61.

55. Norwood R, Balkissoon R. Current perspectives on management of co-morbid depression in COPD. COPD 2005;2:185-93.

56. Li CH, Chen WC, Liao WC, et al. The association between chronic obstructive pulmonary disease and Parkinson's disease: a nationwide population-based retrospective cohort study. QJM 2015;108:39-45.
Cite this article as: Cui Y, Liu KWK, Ip MSM, Liang Y, Mak JCW. Protective effect of selegiline on cigarette smokeinduced oxidative stress and inflammation in rat lungs in vivo. Ann Transl Med 2020;8(21):1418. doi: 10.21037/atm-20-2426 